Classification

Physics Abstracts

$41.10 \mathrm{H}-02.60-03.50$

\title{
Sphères diélectriques non concentriques par MMP-3D
}

\author{
S. Kiener $\left({ }^{1}\right)$ et $M . N e y\left({ }^{2}\right)$ \\ (1) Communications Research Laboratory, Electromagnetic Compatibility division, 4-2-1 \\ Nukui-kitamachi, Koganei, Tokyo 184, Japan \\ $\left({ }^{2}\right)$ Génie Electrique, Université d'Ottawa, 161 Louis Pasteur, Ottawa Ontario, K1N 6N5 \\ Canada
}

(Reçu le 17 mars 1992, accepté le 17 juillet 1992)

\begin{abstract}
Résumé . - Cette communication se propose de présenter rapidement les codes MMP (Multiples MultiPóles) basés sur la TMG (Technique des Multipôles Généralisés) et ensuite de montrer une application spécifique. La diffraction d'une onde plane sur des sphères diélectriques en résonance avec ou sans pertes est un problème classique et qui possède une solution analytique. En revanche, si l'on cherche à calculer à généraliser le problème avec des sphères non concentriques, un formalisme complexe et quelques approximations sont nécessaires. Ce problème peut être calculés par les codes MMP-3D. On s'attachera aussi à valider les résultats d'une manière interne en utilisant les possibilités du code ainsi que d'une manière externe en comparant avec les séries de Mie, mais aussi avec les résultats obtenus par une autre méthode numérique.
\end{abstract}

\begin{abstract}
The aim of this communication is to present shortly the well known MMP codes (Multiple Multipoles Programs) based on the GMT (Generalized Multipole Technique). A specific application is also computed. The scattering of a plane wave on spherical dielectric resonators is a classical problem with an analytic solution. For non concentric spheres, the analytic computation requires a "heavy" formalism and some approximations. However, this problem can be computed with the MMP-3D codes. Great care will be given to the self validation process of the computation with special features belonging to the code as well as external validation which is given, in the case of the single sphere, by the Mie's series and for the non concentric spheres by results computed with another numerical method.
\end{abstract}

\section{Introduction.}

Les sphères diélectriques sont d'usage fréquent aussi bien en optique qu'en micro-ondes. En optique, la diffraction de la lumière sur des particules revient souvent à étudier la diffraction sur des sphères dont de nombreux exemples existent sous une forme approchée dans la nature (gouttelettes d'eau ou d'huile) [2]. 
Pour la construction de circuits intégrés, les résonateurs diélectriques sont couramment utilisés afin d'obtenir un oscillateur avec un facteur $Q$ de grande dimension (plusieurs dizaine de milliers) [1]. L'utilisation peut être un filtre pour les fréquences de résonance.

Ces deux applications sont bien différentes : la longueur d'onde de la lumière visible est de l'ordre de plusieurs milliers d'angströms (fréquence de l'ordre de $500 \mathrm{THz}$ ), alors que les fréquences typiques pour les circuits micro-ondes est de l'ordre 2 à $30 \mathrm{GHz}$. Mais, les particules, dans le cas de l'optique, et les résonateurs diélectriques sont de dimensions avoisinant les 2 longueurs d'onde de la source. On peut donc résoudre deux types de problèmes très différents avec le même modèle.

Il existe encore d'autres applications comme l'étude de l'influence du rayonnement électromagnétique sur le corps humain. Les fréquences sont encore plus basses, mais les dimensions des objets sont plus grandes et on revient sensiblement au même problème que précédemment.

L'ajout d'une seconde sphère sert à représenter, dans une première approximation, le cas d'une sphère diélectrique non linéaire.

\section{Introduction à la méthode.}

Les codes MMP ont été développés à l'Ecole Polytechnique Fédérale de Zurich [3,5], TMG est le nom générique utilisée par différents groupes utilisant les concepts suivants [6-8]:

2.1 Développement direct des champs électriques et magnétiques en série de fonctions de base. En $3 \mathrm{D}$, il suffit de développer la composante radiale:

$$
\begin{aligned}
r E_{\mathrm{ir}} & =A_{\mathrm{i} 0} f_{\mathrm{i} 0}+\sum_{n=1}^{N} A_{\mathrm{in}} f_{\mathrm{in}} \\
\text { avec } \mathrm{H}_{\mathrm{i}} f_{\text {in }} & =0 \text { et } \mathrm{H}_{\mathrm{i}} f_{\mathrm{i} 0}=g_{\mathrm{i}}
\end{aligned}
$$

où $H_{\mathrm{i}}$ est l'opérateur d'Helmholtz et $g_{\mathrm{i}}$ la source (fonction d'inhomogénéité).

2.2 Les fonctions de base $f_{1 n}$ sont des solutions analytiques des équations de champs. En 3D (coordonnées sphériques), les trois principaux types de fonction de base utilisés sont:

i) les ondes planes;

ii) les multipôles dont la forme typique est la suivante :

$$
f_{i n}(r, \theta, \phi)=\sum_{n=0}^{N} \sum_{m=0}^{M} \sqrt{\frac{\pi}{2 k_{\mathrm{i}} r}} H_{n+1 / 2}^{(1)}\left(k_{\mathrm{i}} r\right) P_{n}^{m}(\cos \theta) \cos m \phi
$$

où $H_{n+1 / 2}^{(1)}$ est une fonction de Hankel du premier ordre et $P_{n}^{m}$ un polynôme de Legendre

Le comportement de ces fonctions est essentiellement local (c'est à dire que leur valeur est grande dans le voisinage de leur origine et décroît exponentiellement avec l'éloignement);

iii) et finalement "l'expansion normale", utilisée essentiellement pour le calcul de matériau diélectrique au contour lisse, dont la formulation est la même que celle d'un multipôle en remplaçant les fonctions de Hankel par des fonctions de Bessel.

2.3 Application d'une technique de collocation (Point Matching). - Cette technique est généralisée en utilisant un système surdéterminé d'équations (plus d'équations que d'inconnues) résolu par la méthode des moindres carrés. Soit $P_{l}$ un point du pourtour séparant les domaines $D_{i}$ et $D_{j}$, les conditions de continuités peuvent s'écrire: 


$$
\begin{aligned}
\omega_{\mathrm{user}} \sqrt{d_{l}}\left[E_{t i}\left(\mathrm{P}_{l}\right)-E_{t j}\left(\mathrm{P}_{l}\right)\right] & =\eta_{e t}, \\
\omega_{\text {user }} \sqrt{d_{l}}\left[E_{\tau i}\left(\mathrm{P}_{l}\right)-E_{\tau j}\left(\mathrm{P}_{l}\right)\right] & =\eta_{e \tau}, \\
\omega_{\mathrm{user}} \sqrt{d_{l}} \sqrt{Z_{i} Z_{j}}\left[\mathrm{H}_{t i}\left(\mathrm{P}_{l}\right)-\mathrm{H}_{t j}\left(\mathrm{P}_{l}\right)\right] & =\eta_{h t}, \\
\omega_{\text {user }} \sqrt{d_{l}} \sqrt{Z_{i} Z_{j}}\left[\mathrm{H}_{\tau i}\left(\mathrm{P}_{l}\right)--\mathrm{H}_{\tau j}\left(\mathrm{P}_{l}\right)\right] & =\eta_{h \tau}, \\
\omega_{\mathrm{user}} \sqrt{d_{l}} \epsilon_{i j}^{-1}\left[D_{n i}\left(\mathrm{P}_{l}\right)--D_{n j}\left(\mathrm{P}_{l}\right)\right] & =\eta_{d n}, \\
\omega_{\text {user }} \sqrt{d_{l}} \mu_{i j}^{-1} Z_{i} Z_{j}\left[B_{n i}\left(\mathrm{P}_{l}\right)-B_{n j}\left(\mathrm{P}_{l}\right)\right] & =\eta_{b n}, \\
\sum \eta_{i}^{2} & =\min .
\end{aligned}
$$

où les indices $t$ et $\tau$ indique deux directions tangentes et $n$ la direction normale au pourtour au point $P_{l}$. On remarque que les six conditions de continuité sont utilisées, malgré le fait qu'elles soient linéairement dépendantes. On a aussi soigneusement normé chaque équation afin que leurs dimensions soient semblables.

Pour de nombreuses méthodes numériques, la sphère diélectrique n'est pas une géométrie favorable. En particulier, pour toutes les méthodes qui doivent discrétiser l'intérieur de chaque matériau à l'aide d'éléments cubiques ou pyramidaux.

En revanche pour les codes MMP-3D, cette géométrie paraît, a priori, bien adaptée. Les fonctions de base sont des fonctions sphériques et seul le pourtour du corps doit être discrétiser.

\section{Le problème de la sphère diélectrique en résonnance.}

Comme il l'a été précisé dans l'introduction, il existe une solution analytique pour une seule sphère diélectrique par les séries de Mie ou d'une manière équivalente par les équations aux valeurs propres de Debye. On rappelle que la condition de résonance est décrite par les relations suivantes [1]:

$$
\begin{aligned}
& \mathrm{TM}: \frac{J_{n-1 / 2}\left(k_{\mathrm{d}} r\right)}{J_{n+1 / 2}\left(k_{\mathrm{d}} r\right)}=\sqrt{\epsilon_{\mathrm{R}}} \frac{H_{n-1 / 2}^{(2)}\left(k_{0} r\right)}{H_{n+1 / 2}^{(2)}\left(k_{0} r\right)}-n \frac{\epsilon_{\mathrm{R}}-1}{k_{\mathrm{d}} r} \\
& \mathrm{TE}: \frac{J_{n-1 / 2}\left(k_{\mathrm{d}} r\right)}{J_{n+1 / 2}\left(k_{\mathrm{d}} r\right)}=\frac{1}{\sqrt{\epsilon_{\mathrm{R}}}} \frac{H_{n-1 / 2}^{(2)}\left(k_{0} r\right)}{H_{n+1 / 2}^{(2)}\left(k_{0} r\right)}
\end{aligned}
$$

où $k_{\mathrm{d}}$ et $k_{0}$ sont les nombres vectoriels à l'intérieur de la sphère et dans le vide, $\epsilon_{\mathbf{R}}$ la permittivité relative de la sphère (constante réelle), $H_{\nu}$ et $J_{\nu}$ sont respectivement des fonctions de Hankel et de Bessel.

Le propos n'est pas de calculer les fréquences de résonance de la sphère diélectrique, mais de rappeler les conditions pour lesquelles cette résonance intervient.

Pour plusieurs sphères non concentriques, le problème peut encore se résoudre d'une manière analytique à l'aide du théorème d'addition pour les fonctions d'ondes sphèriques [4]. Mais la réalisation du calcul impose certaines conditions limitatives aux valeurs des indices de réfraction des sphères qui doivent être très proches (calcul de perturbation). 


\section{Calcul de sphères diélectriques avec MMP-3D.}

On remarquera dans les principes énoncés précédemment (Sect. 2.2) que les fonctions multipôles ont une singularité à l'origine. En revanche, l'expansion normale avec des fonctions de Bessel ne présente qu'une singularité à l'infini, si la conductivité n'est pas nulle.

Pour la modélisation avec le code MMP du champ électromagnétique d'une sphère diélectrique, il suffit de placer un seul développement en fonctions de Bessel (expansion normale dont le nombre de coefficients inconnus dépend de la fréquence) au centre de la sphère diélectrique pour le champ à l'intérieur de la sphère, un développement en fonctions de Hankel (multipôle avec le même nombre de coefficients et la même origine que l'expansion normale) et une onde plane pour modeler le champ à l'extérieur de la sphère.

On applique ensuite la collocation généralisée, présentée dans le paragraphe 2.3 , en un certain nombre de points (MP) répartis sur le pourtour de la sphère.

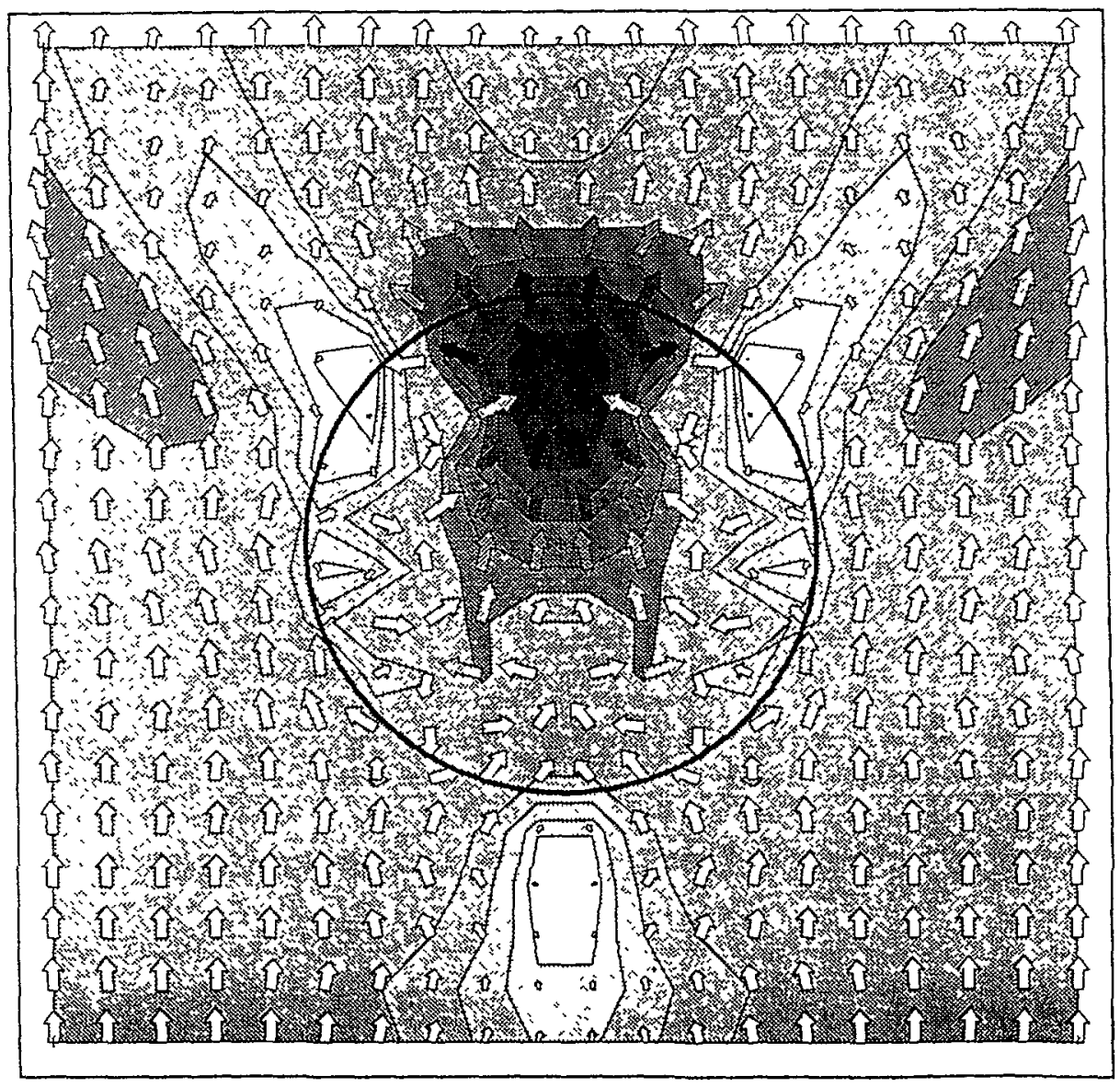

Fig. 1. - Vecteur de Poynting moyen: existence de points chauds pour une sphère de $26 \mathrm{~cm}$ de diamètre $\left(\epsilon_{R}=4\right)$, illuminée par une onde plane sinusoïdale de fréquence $1,5 \mathrm{GHz}$.

[Average Poynting vector: "Hotspots" for a sphere of $26 \mathrm{~cm}$ diameter $\left(\epsilon_{R}=4\right)$. The source is a sinusoidal plane wave of $1.5 \mathrm{GHz}$ frequency.] 
Les codes MMP-3D ne se servent pas des propriétés de symmétrie de révolution des corps à calculer. Seules les symmétries diadiques par rapport aux trois plans de base sont utilisées. Dans notre cas, la géométrie totale se réduit à $1 / 8$ ème. L'efficacité de la méthode est directement dépendante du nombre de MP nécessaire au calcul du problème. La règle générale dicte qu'il faut prendre de 5 à 10 MP par longueur d'onde ce qui donne le nombre d'équations à résoudre. Pour des géométries aussi favorable que la sphère, on peut se contenter du minimum de MP. Ensuite, le processus standard consiste à diviser le nombre d'équations par un facteur qui peut varier de 2 à 10 (facteur de surdétermination) pour obtenir le nombre de coefficients inconnus. Les ordres $n_{\max }$ et $m_{\max }$ sont finalement déduits à l'aide de simples considérations géométriques $[5,7]$.

Ce processus est parfaitement efficace pour des sphères qui ne sont pas en résonance comme le montre la figure 1. Ce problème se calcule en quelques secondes sur un PC standard équipé d'un processeur Intel $80386+80387$.

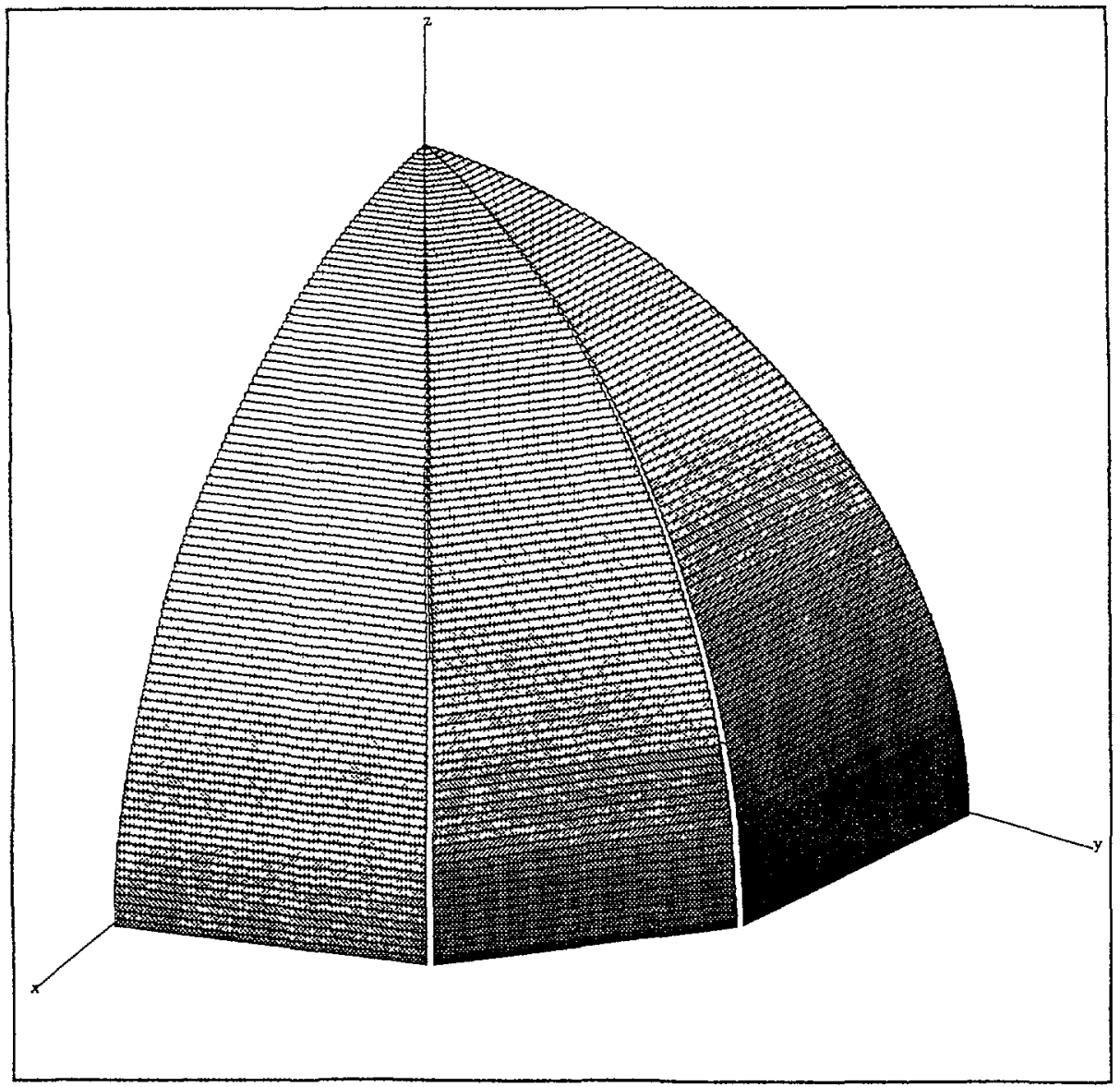

Fig. 2. - Répartition de l'erreur sur les MP de la sphère en résonnance en utilisant le processus standard de calcul.

[Error distribution on the resonant sphere's MP using the standard method.] 
En revanche, les formules (1) et (2) montrent qu'à chaque fréquence de résonance correspond un ordre pour les fonctions sphériques. Avec le procédé standard et pour les hautes fréquences, cet ordre risque de ne pas ètre obtenu et le résultat devient tout à fait médiocre (Fig. 2).

Cela est dû au fait que ce problème est un problème de valeurs propres qui impliquerait de chercher les valeurs propres du système d'équations. En choisissant bien le mode dominant, on trouve exactement l'équation (2).

Pour obtenir un résultat à nouveau correct, il existe deux méthodes approchées pour résoudre ce problème: une solution empirique consiste à augmenter le nombre de MP (et donc l'ordre des fonctions de base) jusqu'à obtenir des résultats satisfaisants. L'inconvénient provient de l'augmentation importante du temps de calcul, mais c'est un processus simple et direct.

L'autre solution consiste à calculer les ordres nécessaires à l'aide des formules (1) et (2) et d'injecter essentiellement ces ordres dans le multipôle et l'expansion normale. Le deuxième cas implique la connaissance à priori des fréquences de résonances. C'est effectivement directement applicable dans le cas de la géométrie présentée, mais pas pour d'autres formes.

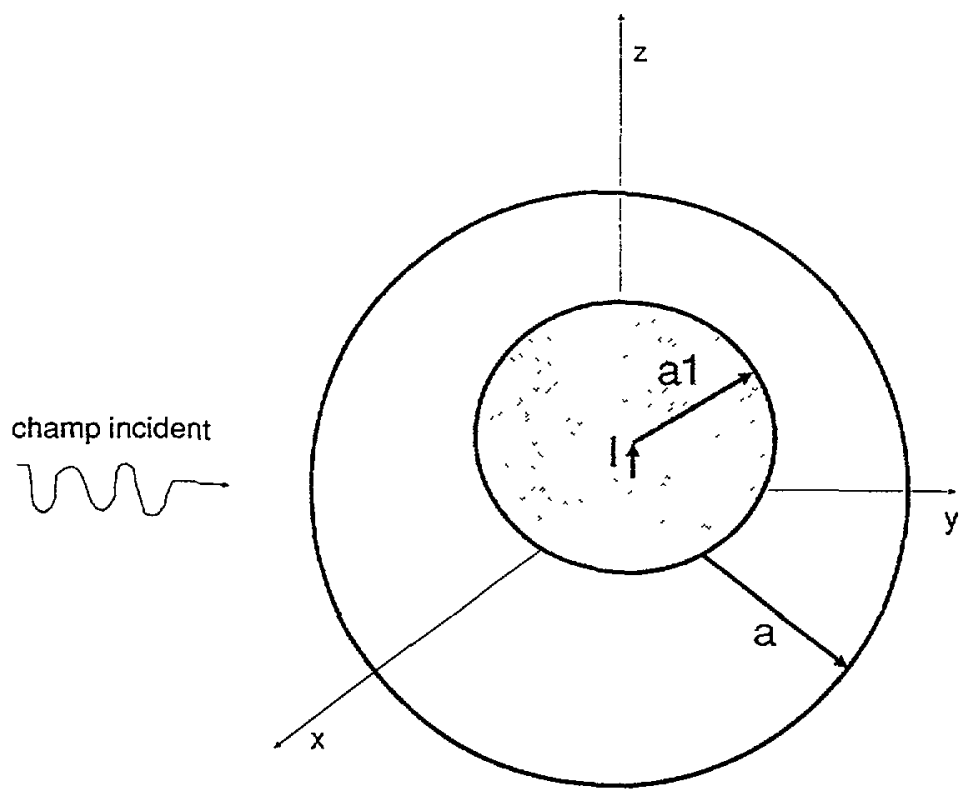

Fig. 3. - Géométrie de deux sphères non-concentriques: $a=$ rayon de la grande sphère $\left(\epsilon_{\mathrm{R}}=4\right)$, $a 1=$ rayon de la petite sphere $\left(\epsilon_{R}=4,441 \ldots\right), l=$ distance entre les centres des sphères.

[Geometry of two non-concentric spheres: $a=$ radius of the big sphere $\left(\epsilon_{\mathrm{R}}=4\right), a 1=$ radius of the small sphere $\left(\epsilon_{\mathrm{R}}=4.441\right.$. $), l=$ distance between the center of the spheres.]

Finalement, pour calculer la géométrie avec les sphères non concentriques (Fig. 3), on applique les mêmes principes, sans se préoccuper le moins du monde du couplage entre les deux sphères qui est implicitement inclus dans le calcul.

Le temps de calcul est proportionnel au produit du nombre des équations par le nombre d'inconnues au carré. La figure 4 montre le résultat du calcul en représentant la SER (Surface équivalente radar) en fonction de différents fréquences (ou plutôt du paramètre de dimension $k a, a$ étant le rayon de la sphère). 


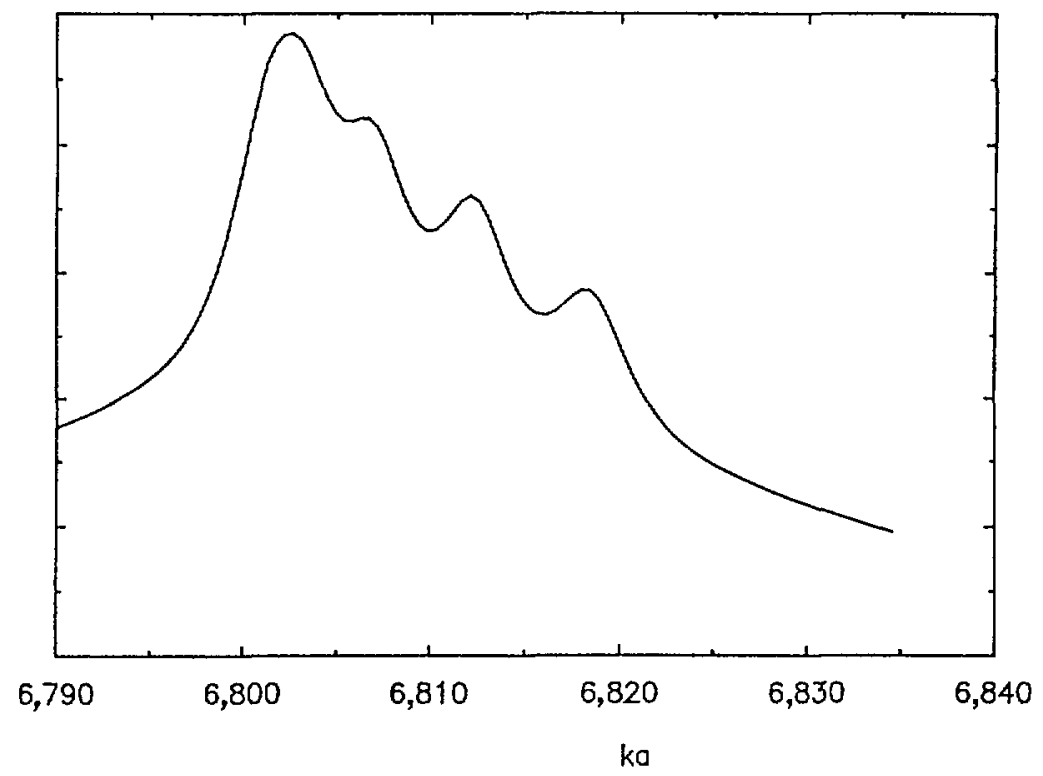

Fig. 4. - Surface équivalente radar (SER) pour les sphères de la figure 3.

[Radar Cross Section (RCS) for the figure 3 geometry.]

La validation s'effectue en contrôlant la convergence des coefficients, l'erreur sur le pourtour (diffèrence entre le champ tangent intérieur et extérieur), ainsi que les calculs en champ lointain où la SER (surface équivalente radar) ne doit pas montrer de polarisation croisée.

De plus, cette courbe a été confirmé par les résultats obtenus avec la T-Matrix [2 et communication privée].

\section{Conclusions.}

L'exemple de sphères diélectriques en résonance peut être calculé en tenant compte des propriétés des fréquences de résonance par les codes MMP-3D. Pour des sphères excentriques, il est nécessaire d'utiliser une méthode numérique bien adaptée à ce type de géométrie.

Ceci est particulièrement important si les sphères sont électriquement grandes, comme c'est le cas ici, et que la dimension du problème matriciel est souvent une fonction cubique de la fréquence.

Du fait de ses nombreuses applications, ce type de géométrie pourrait aussi servir d'exemple canonique dans le cadre d'une bibliothèque d'applications pour les hautes fréquences.

\section{Bibliographie}

[1] AfFolter P. and ElLIASSON B., Electromagnetic Resonances and Q-Factors of Lossy Dielectric Spheres, IEEE Trans. MTT, MTT 21 No. 9 (September 1973).

[2] Barber P. W. and Hill S. C., Light Scattering by Particles: Computational Methods, Advanced Series in Applied Physics, (World Scientific, 1990). 
[3] Bomholt L., MMP3D: A Computer Code for Electromagnetic Scattering Based on the GMT, Thesis ETHZ, (Zurich, 1990).

[4] Fikioris J. G. and UzUnOglu N. K., Scattering from an Eccentrically Stratified Dielectric Sphere, J. Opt. Soc. Am. 69 No. 10 (October 1979).

[5] HaFNER Ch. V., The Generalized Multipole Technique for computational Electromagnetics (Artech House, July 90).

[6] HAFNer Ch. V., On the Relations of MoM and GMT, IEEE AP's Magazine (1991).

[7] Kiener S., Introduction aux codes MMP et à la TMG, Rev. Phys. Appl. 25 (Modélisation en électromagnétisme) (1990) 737-749.

[8] Ludwig A., A new technique for numerical electromagnetics, IEEE AP's Newslett. 31 (1989) 40-41. 
Articles Réguliers 